\title{
VERZEICHNIS ZUM ZWÖLFTEN JAHRGANG
}

\section{Jänner}

Osterreichische Kunstpreisträger . . . . . . . . . . . . . . . . . . . . . 1

Dr. Wilhelm Ki enz l $\mathbf{t}$ : "Wer soll Meister sein?" . . . . . . . . . . . . 3

Prof. André Es pị a u de la Maestre: Milhauds "Christophe Colomb". . . 9

Prof. Dr. Ernst T it t e 1: Musiculs und Cantor . . . . . . . . . . . . . 13

Dr. Alexander We i n m a n : Eine Plagiatbeschuldigung gegen Schubert . . 19

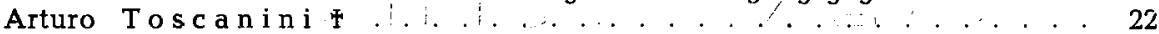

Prof. Erik W e r b a : Komponistennachwuchs (Nachlese zum Wettbewerb der OMZ) . . . . . . . . . . . . . . . . . . . . . . . 23

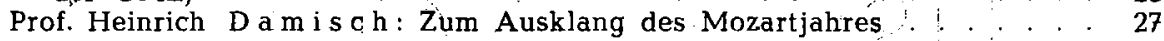

\section{Februar}

Prof. Dr. H. H. S t u ckenschmidt: Moderne Psalmen von Arnold Schönberg . . . . . . . . . . . . . . . . . . . . . . . . . . . . . . 47

Jenö v. Ta k á c s: Béla Bartók in Osterreich . . . . . . . 50

Prof. Dr. K. H. R u p p e 1: Carl Orffs "Trionfi" . . .. . . . . . . . . . . . . . 54

Dr. Erika He lle I : Zur Technik und Ästhetik des Bekanto . . . . . . . . 59

Pro. Dr. Erich H. M ülle r v. A s ow : Ein ungedruckter Brief Mahlers . . . 63

Hanns W i $\mathrm{t}$ e $\mathrm{r}$ : Hans Erich Apostel . . . . . . . . . . . . . . . . . . 65

Präsident Dr. Hans Sittner: Otto Siegel . . . . . . . . . . . . 67

Wina Morelli-Ga ll et: Das Museo teatrale alla Scala . . . . . . 69

Dr. Gustav P i ch le r: Salzburger Mozartfeier 1957. . . . . . . . . . . 71

Ludwig Z a $\mathbf{n}$ : Aus der Musiksammlung der Stadt Wien . . . . . . . . 72

Josef Friedrich Doppelbauer: Der Welser Bach-Chor . . . . . . 73

Gotthard Feustel: Gedanken über Mozart und Wagner 75

März

Prof. Max Unger: Vom geselligen Beethoven .. . . . . . . . . . . . . . 91

Dr. Kurt Pa h len: Manuel de Falla und die Musik in Spanien $\quad . \quad$. 101

Rudolf Kl e in : Johann Nepomuk David . . . . . . . . . . . . . . . . 106

Dr. Johannes S c h w i e ger: Josef Mathias Hauer . . . . . . . . . . . . 108

Univ.-Prof. Hofrat Dr. Leopold Now a $k$ : Das Archiv für Phonogramme musikalischer Handschriften . . . . . . . . . . . . . . . . . . . . . 110

Dr. Rudolf R e t i F: Die Entstehung der IGNM . . . . . . . . . . . . . . . . . 113

Dr. Norbert Ts ch u lik: Wird das'Theater an der Wien reaktiviert? . . . 117

Dr. Kurt G. R o ge r: Prokofieffs "Krieg und Frieden“ als Fernsehoper . . . 119

April

Prof. Hans S wa r o w s k y : Persönliches von Richard Strauss (1) . . . . . 137

Prof. Josef Mer $\mathrm{t}$ i $\mathrm{n}$ : Uber das Cembalo . . . . . . . . . . . . . . . . . 142

Dr. Paul L o r e n z: Schopenhauer als Wagner-Kritiker . . . . . . . . . . . 149

Rudolf Kle i $\mathrm{n}$ : Molière auf der Opernbühne . . . . . . . . . . . . . . 151

Kurt B la u k o p f: Balzac im elektronischen Studio . . . . . . . . . . . 154

Drei Variationen über das Thema "Romantischer Realist"

(Joseph Marx) . . . . . . . . . . . . . . . . . . . . . . . . . . . 158

Präsident Dr. Bernhard P a umgart $\mathrm{m}$ er: Egon Kornauth . . . . . . . . 160

Vor dem 8. Wiener Musikfest . . . . . . . . . . . . . . . . . . . . . . 162

Prof. Dr. Otto E. D e ut s c h: Zum "geselligen Beethoven" . . . . . . . . 163

Ludwig Z a n t: Sänger und Komponisten in italienischen Karrikaturen . . . 164

Dr. Dolf L i n d n e r : "Pallas Athene weint" in Linz . . . . . . . . . . . . 166

\section{Mai}

Prof. Dr. Otto E. Deut s ch : Rossinis Lebewohl an die Wiener . . . . . . 181

Robbins H. C. L a n d o $n$ : Eine aufgefundene Haydn-Messe . . . . . . . . . . 183

Prof. Hans S wa r o w s k y : Persönliches von Richard Strauss (II) . . . . . 186

Prof. Dr. Erik W e r b a : Ein mährisches Liederbuch . . . . . . . . . . . 191

Prof. Dr. Helmut A. Fi e c h t n e r : Béla Bartók . . . . . . . . . . . . . . 193

Dr. Hans J a n č i k: Ernst Tittel . . . . . . . : . . . . . . . . . . . . . . . . . 197

Dr. Dolf Li $\mathrm{n}$ d n e r: Anton Heiller . . . . . . . . . . . . . . . . . . . . . 199 
Sektionschef Dr. Egon H i l b e r t: Ensemble, Budget und Spielplan . . . . 202

Rudolf Klein: "Othello" unter Karajan . . . . . . . . . . . . . . . 204

Claus-Henning B a ch man $n$ : Alban-Berg-Tage in Hamburg . . . . . . . 206

Konzert der Preisträger des Wettbewerbes der OMZ . . . . . . . . . 214

Juni

Friedrich Gulda: Jazz und wir. . . . . . . . . . . . . . . . . . . . : 233

prof. Dr. Egon Komorzy n k i : Ist Papageno ein "Hanswurst"? . . . 225

Dr. Ernst P a u l: Osterreichs Jagdmusik . . . . . . . . . . . . . . . . . 230

Dr. Gustav Pi ch le r : Ein Doppelgänger des "Hobelliedes" . . . . . 237

Prof. Max S chön h er r: Wie ein Wiener Ballett entstand . . . . . 240

Prof. Dr. Erik Werba : Walter Andress. . . . . . . . . . . . . . . 244

Dr. Rudolph Franz B r a u n e r : Armin Kaufmann . . . . . . . . . . . . 246

Rudolf Os ter reicher: Aus der Glanzzeit der Wiener Operette . . . . 249

General Anton L e há $\mathrm{r}$ : Das Lehár-Schikanender-Schlößl in Wien-Nußdorf 254

Luis Grundner : Präsident Puthon - 85 Jahre alt . . . . . . . . . . . 256

Dr. Dolf L i n d n e r: Enrico Mainardi zum 60. Geburtstag . . . . . . . . . 256

Rudolf Klein: Musica viva in Zürich . . . . . . . . . . . . 258

\section{Juli-August}

Yehudi Menuhin: Wir und die Musik . . . . . . . . . . . . . . . . . . 271

Christopher Ra eburn: "Figaro " in Wien . . . . . . . . . . . . . . . . 273

Prof. Dr. Otto E. D e u t s c h: Zur "Kleinen Freimaurerkantate" . . . 277

Serge Kous s evitzky: Vom schöpferischen Dirigieren . . . . . . . . 281

Walter Berry: Die Schule-der Sänger . . . . . . . . . . . . . . . . . . 285

Prof. Dr. Siegfried B or ris: Grundtypen des musikalischen Erlebens . . . 287

Das neue Salzburger Festspielhaus . . . . . . . . . . . . . . . . . . . . 293

Prof. Dr. Helmut A. F i e c h t n e r: Theodor Berger . . . . . . . . . . . . . 294

Dr. Alexander Wi tes ch n ik: Alfred Uhl . . . . . . . . . . . . . . . 296

Prof Franz L e d w i n k a : Der Mozart-Schüler Heribert v. Karajan . . . . 298

Prof. Heinrich D a m is ch: Die ersten Salzburger Kritiken über Heribert von Karajan . . . . . . . . . . . . . . . . . . . . . . . . . . . . . 300

Alfred B ren de I: Der Wachtraum vom Jazz . . . . . . . . . . . . . . . . 302

Prof. Dr. Erik Werba, Rudolf Klein, Dr. Dolf L ind ner,

Prof. Dr. Heinrich Ne u m a e r: Die Bilanz der Wiener Festwochen . 305

Prof. Karl Heinz D w o r c z a k : Grazer Sommerspiele 1957 . . . . . . . . 310

Kapfenberger Kulturtage 1957 . . . . . . . . . . . . . . . . . . . . . . 311

Claus-Henning B a ch m a n n: Große Dichtung als Oper . . . . . . . . 312

Dr. Ludwig K. M a y e I: Gogols "Revisor" als Oper . . . . . . . . . . . . . 314

Prof. Dr. Walter Koln e d e r : Wiener Initiative für Haarlem . . . . . . . 315

Prof. Dr. Roland Ten s che r t : 100 Jahre Musikhaus Doblinger . . . 316

\section{September}

Christopher Ra eburn: Das Zeitmaß in Mozarts Opern . . . . . . . . . 329

Alfred B r e n d e 1: Zwischen Kompetenz und Willkür . . . . . . . . . . . 333

Dr. Carl Nemeth : Ein neuaufgefundenes Skizzenblatt zu Franz Schmidts

"Variationen über ein Husarenlied" . . . . . . . . . . . . . . . . . 336

Dr. Gustav P i ch le r: Der "Chopin der Nordens" . . . . . . . . . . 340

Prof. Ewald Rudolf C w i e $\mathrm{n} \mathrm{k}$ : Ernst Ludwig Uray . . . . . . . . . . . . . 342

Dr. Manfred B l u m a u e r : Max Hager . . . . . . . . . . . . . . . . . . . 344

Salzburger Festspiele 1957

Prof. Dr. Erik Wer b a : Von Mozart zu Richard Strauss . . . . . . . 346

Dr. Gustav Pi c h le r : Konzerte und Liederabende . . . . . . . . . . 349

Rudolf Kle i n : "Die Schule der Frauen" . . . . . . . . . . . . . . . 350

Oswald Lutz: Bregenzer Festspiele 1957 . . . . . . . . . . . . . . . . 352

Dr. Dolf L in d n e r: Musik im sommerlichen Wien . . . . . . . . . . . . . 354

Dr. Wilfried Scheib: Akademie-Kammerchor vor "Internationalem

Forum" . . . . . . . . . . . . . . . . . . . . . . . . . . . . . . . 355

Rudolf Kl e in: "Ein belämmerndes Ereignis in Seldwyla" . . . . . . . . . . 358

Dr. Robert B r e u e r : Wird die Fernsehoper die Oper "retten ${ }^{\Perp}$ ? . . . . . . 360

Karl Löb l: Zur Uraufführung von Hindemiths "Harmonie der Welt" . . . 361

E. I. L u i n: Florentiner Musikwochen . . . . . . . . . . . . . . . . . 362 


\section{Oktober}

Bruno W a lter Das Zeitelement in der Oper . . . . . . . . . 373

Dr. H. C. Robbins L a n d o n: Ein neuentdecktes Bildnis Joseph Haydns : : 381

Prof. Dr. Otto Erich Deuts ch: Die Orangerie im Schloß Schönbrunn . . 384

Kim B or g: Jean Sibelius und die Finnen . . . . . . . . . . . . . 386

Franz K. M üller: Gesamtverzeichnis der Werke von Jean Sibelius. . . . 390

Franz M a ille r : Karl Schiske . . . . . . . . . . . . . . . . 395

Dr. Erwin R i e g e r: Karl Etti . . . . . . . . . . . . . . . . . . . . . . 397

Prof. Dr. Hans S it t n e r: Pianistenwettbewerb in Brasilien. . . . . . . . 399

Hofrat Dr. Wilhelm Wald st e in : Bayreuth $1957 \ldots . . . . . . . .401$

Claus-Henning B a ch ma n n : Neue Musik auf der Biennale . . . . . . 404

Dr. Paul L. o r e n z: Gustav Mahler — ein Unvergessener . . . . . . . . . 405

Dr. Franz Willna uer: Osterreichischer Komponistennachwuchs bei den

Hochschulwochen in Alpbach . . . . . . . . . . . . . . 407

Prof. Dr. Walter $\mathrm{Hummel}$ : Jubiläen der Musikwissenschaftlichen

Tagungen am Mozarteum . . . . . . . . . . . . . . . . . . . . . 408

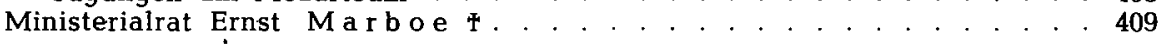

\section{November}

Kurt P a hle n: Das Teatro Colón . . . . . . . . . . . . . . . . 419

Dr. Dolf Li n d n e r : Der Tanz als Ausdruck der Zeit . . . . . . . . . 423

Karl Heinz Fü s s 1: "Medusa“, das jüngste Ballett von G. v. Einem . . . . 431

Dr. Herbert Vog g: Fritz Skorzeny . . . . . . . . . . . . . . . . . 434

Hofrat Dr. Wilhelm W a $1 \mathrm{~d}$ s te in : Leopold Matthias Walzel . . . . . . 436

Prof. Dr. Egon Korna $\mathrm{th}$ : Bernhard Paumgartner zum 70. Geburtstag . . 438

Dr. Rudolf B a u e r: Moral und Amoral am Klavier. Alfred Cortot zum

80. Geburtstag . . . . . . . 443

Prof. Dr. Erik We r b a : Wilhelm Waldstein sechzig Jahre alt . . . . . . 445

Konstantin Prod'homme: Die Tradition des Nichtherkömmlichen.

Der Anteil der Musik an den Berliner Festwochen 1957 . . . . . . . . . 446

Rudolf Kle i $\mathrm{n}$ : Donaueschingen 1957 . . . . . . . . . . . . . . . . . . 449

Dr. E. I. L u i n : Wiener Chöre bei der Sagra Musicale Umbra . . . . . . . 451

Dr. Heinrich St u de r: In memoriam Karl Kobald . . . . . . . . . . . . 452

Internationale Musikakademie im Osterreich-Pavillon der

Weltausstellung in Brüssel .................. . . 453

Isolde R i e h 1: David-Uraufführung in Stuttgart . . . . . . . . . . . 454

\section{Dezember}

Dr. Hedwig M. v. As o w: Weihnachtliche Musiker-Briefe . . . . . . . . 464

Prof. Dr. Hans Joachim Moser: Weihnachtsmusik der deutschen

Landschaft . . . . . . . . . . . . . . . . . . . . . . . . . . . 472

Dr. Alexander W e inma n : Zur Geschichte des Alt-Wiener Musikverlages . . . . . . . . . . . . . . . . . . : 474

Karl Franz M ü 1 le r : Maurice Ravel (mit Werksverzeichnis) . . . . . 481

Prof. Dr. Franz Ko s c h: Erich Romanovsky . . . . . . . . . . . . . 487

Hofrat Univ.-Prof. Dr. Leopold N o wa k: "Haydn - HoV“ . . . . . . . . 489

Hofrat Prof. Dr. Viktor Keld orfer: Ewiger Urheber- und Denkmalschutz 490

Rudolf Kl ei n: Paul Wittgenstein zum 70. Geburtstag . . . . . . . 494

\section{Jänner-Dezember}

Kritik einst und jetzt 32, 76, 121, 167, 207

Osterreichische Chronik 33, 77, 124, 169, 208, 363, 410, 455, 496

Literatur $37,80,127,174,211,364,460,502$

Neue Schallplatten $39,173,365$

Nachrichten 40, 85, 129, 175, 215, 366, 412, 459, 499

Aus den österreichischen Musikinstituten:

Akademie Wien 42, 88, 134, 177, 220, 413, 461, 503

Konservatorium der Stadt Wien 43,88, 135, 179, 221, 371, 413, 361, 505

Mozarteum Salzburg 44,84, 136, 179, 369, 413, 461, 506

Konservatorium Innsbruck 414 